\title{
Discovery of a [WO] central star in the planetary nebula Th 2-A (Research Note)
}

\author{
W. A. Weidmann ${ }^{1,2}$, R. Gamen ${ }^{2,3}$, R. J. Díaz ${ }^{3,4}$, and V. S. Niemela ${ }^{5}$ \\ ${ }^{1}$ Observatorio Astronómico Córdoba, Universidad Nacional de Córdoba, Argentina \\ e-mail: weidmann.walter@gmail.com \\ 2 Consejo Nacional de Investigaciones Científicas y Técnicas, Argentina \\ 3 Complejo Astronómico El Leoncito, Avda. España 1412 Sur, San Juan, Argentina \\ 4 Gemini Observatory, AURA, La Serena, Chile \\ ${ }^{5}$ In memoriam (1936-2006)
}

Received 16 April 2008 / Accepted 30 June 2008

\section{ABSTRACT}

\begin{abstract}
Context. About 2500 planetary nebulae are known in our Galaxy but only 224 have central stars with reported spectral types in the Strasbourg-ESO Catalogue of Galactic Planetary Nebulae (Acker et al. 1992; Acker et al. 1996).

Aims. We have started an observational program aiming to increase the number of PN central stars with spectral classification. Methods. By means of spectroscopy and high resolution imaging, we identify the position and true nature of the central star. We carried out low resolution spectroscopic observations at CASLEO telescope, complemented with medium resolution spectroscopy performed at Gemini South and Magellan telescopes.

Results. As a first outcome of this survey, we present for the first time the spectra of the central star of the PN Th 2-A. These spectra show emission lines of ionized $\mathrm{C}$ and $\mathrm{O}$, typical in Wolf-Rayet stars.

Conclusions. We identify the position of that central star, which is not the brightest one of the visual central pair. We classify it as of type [WO 3]pec, which is consistent with the high excitation and dynamical age of the nebula.
\end{abstract}

Key words. ISM: planetary nebulae individual: PN G306.4-00.6 - stars: Wolf-Rayet

\section{Introduction}

The central stars of planetary nebulae (CSPN) play an important role in the understanding of post-AGB stellar evolution. Planetary Nebulae (PNs) are the relics of intermediate-mass stars, and they are important probes of stellar evolution, stellar populations, and cosmic recycling. Understanding the evolutionary status of particular classes of CSs is relevant in a broader astrophysical sense.

About $60 \%$ of the PNe listed in the Strasbourg - ESO Catalogue of Galactic Planetary Nebulae (Acker et al. 1992; Acker et al. 1996) include data concerning the CSPN, but only $30 \%$ of these actually have spectral classification. Among the 224 spectral types reported in that catalog, there are 75 stars presenting emission lines typical of Wolf-Rayet (WR) stars (Acker \& Neiner 2003, hereafter AN03).

The [WO] type (Oxygen sequence) was introduced by Smith \& Aller (1969) to classify a group of 7 CSPN showing an optical WR-like spectrum with strong OVI emission lines. Subsequently, Barlow \& Hummer (1982) defined WO stars as a new WR subtype. The most prominent feature of these objects is the strong emission of the O VI $\lambda \lambda 3811-34 \AA$ doublet, other strong emission lines in the spectra of [WO] stars are the

\footnotetext{
* Based on data collected at (i) the Complejo Astronómico El Leoncito (CASLEO), which is operated under agreement between the Consejo Nacional de Investigaciones Científicas y Técnicas de la República Argentina y Universidades Nacionales de La Plata, Córdoba y San Juan, Argentina; (ii) the $6.5 \mathrm{~m}$ Magellan Telescopes at Las Campanas Observatory, Chile; (iii) the $8 \mathrm{~m}$ Gemini South Telescope, Chile.
}

C IV + He II $\lambda \lambda 4658-86 \AA$ A blend and C IV $\lambda \lambda 5801-12 \AA$ doublet, features which are also exhibited strongly by early WC stars. At the moment there are only four WO stars known in our Galaxy (van der Hucht 2001; Drew et al. 2004) and about twenty [WO] (AN03; Gesicki et al. 2006).

We have started an observational program at CASLEO with the goal of studying the spectra of hitherto unknown CSPNe. In this paper we present one of our first results, i.e. the discovery of a new [WO] star.

\section{Observations}

The initial observations were carried out on March 21, 2006, using the REOSC spectrograph attached to the $2.15 \mathrm{~m}$ telescope at CASLEO, Argentina. These spectra were taken with a 300 line $\mathrm{mm}^{-1}$ grating, which provides a dispersion of $3.4 \AA \mathrm{px}^{-1}$, and a wavelength range of $3500-7000 \AA$ on a Tek $1024 \times 1024$ pixels CCD.

As will be noted in Sect. 3, the CS of the PN Th 2-A has a visual companion separated by 1". 4 (See Fig. 1), which certainly is marginally resolved from CASLEO. Then, the slit was centered on the stars labeled A and B in the Fig. 1, in the E-W direction with a slit width of $3^{\prime \prime}$. To be sure of the sources identifications in the $(\mathrm{A}+\mathrm{B}) \mathrm{CASLEO}$ spectrum, we observed the A and B stars with the Gemini Multi-Object Spectrograph (GMOS) at Gemini South, Chile (Proposal GS-2007B-Q-251). A long-slit of 0.5 arcsec was placed with the orientation of the line joining the $A$ and B stars. A grating of 600 line $\mathrm{mm}^{-1}$ was used which provided 


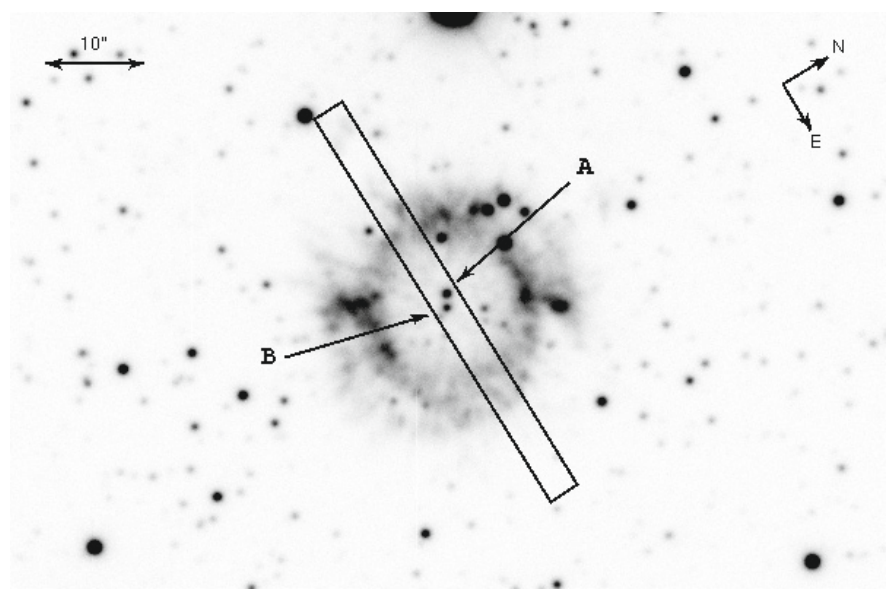

Fig. 1. The PN Th 2-A as seen with GEMINI-South, filter Ha_G0336, with a seeing of 0.7 arcsec. The orientation and size of the CASLEO slit is indicated. The star labeled B is the true and newly identified CS.

a spectral coverage of 3630-6440 $\AA$ and a dispersion of $\sim 0.45$ $\AA \mathrm{px}^{-1}$.

N. Morrell provided us an additional spectrum obtained with the Baade Magellan 6.5-m telescope, at Las Campanas Observatory (LCO) in Chile, during a night with 0.7 arcsec seeing. The Inamori Magellan Areal Camera and Spectrograph (IMACS) was used in its short camera mode, and the grism 20015.0 as dispersor. The observation resulted in a splitted spectrum with a wavelength coverage of 3800-6610 ̊ and 6700-9430 and a dispersion of 1.2 and $1.3 \AA \mathrm{px}^{-1}$ for the blue and red part respectively.

\section{Results and discussion}

The central star of Th 2-A (object A in Fig. 1) was described by Kerber et al. (2003) as being the "well defined photometric center" of the nebula (PHOT in their identification class). Ciardullo et al. (1999) determined its Johnson $V$-band magnitude $V=17.08$ using HST images. They included this PN in their survey for resolved companions, because they noted a nearby companion (star B), which resulted to have a $\sim 50 \%$ probability of being a chance superposition. On the other hand, we did not find any spectroscopic study reported in the bibliography, related with the CSPN of Th 2-A.

The inspection of our $(\mathrm{A}+\mathrm{B})$ CASLEO spectrum revealed that the CSPN Th 2-A was one of the rare [WO] stars, thus claiming our attention. Searching for published information about this star, we found the above cited Ciardullo et al.'s work and retrieved higher spatial resolution HST-WFPC2 images from the MAST archive. We noted that certainly the B star seems more centered in the nebula than A. Moreover, we performed a preliminary aperture-photometry, and estimated that the B star is bluer than A. But, in order to get confidence about the true identification of the central star of the PN, we obtained a high spatial resolution spectrum along the A and B stars (see Fig. 1). Then, the GMOS-S spectrum (see observation description) confirmed that the B star is in fact the CSPN and A star is a late type one. These high spatial resolution images and spectra will be presented and discussed elsewhere in a thorough analysis of the whole planetary nebula system.

In our optical spectra of star B, we identified broad and intense C IV and O VI emission lines, that are typical of WR stars, thus confirming that this spectrum was originated in the nucleus
Table 1. Emission lines identified in the LCO spectrum of the central star of the PN Th 2-A over the 3520-9430 $\AA$ wavelength range. First column shows the central wavelength measured in our spectrum, when a deblend was not possible the centroid of the blend is indicated. The marks ":" and "n.m." refers to uncertain values and values no measured respectively.

\begin{tabular}{|c|c|c|c|}
\hline Line $[\AA ̊]$ & Ion & FWHM $[\AA]$ & $W_{\lambda}[\AA]$ \\
\hline 3822 & O VI & 80 & 580 \\
\hline 4118 & $\mathrm{OV}$ & 31 & 67 \\
\hline 4222 & CIV? & 58 & 22 \\
\hline 4342 & He II, C IV & 40 & 20 \\
\hline 4515 & O VI,O V & 101 & 44 \\
\hline 4674 & C IV,He II & 66 & 385 \\
\hline 4856 & He II, C IV & 50 & 9 \\
\hline 5101 & Ov? & 9 & 71 \\
\hline 5287 & O VI & 54 & 33 \\
\hline 5413 & C IV, He II & 88 & 59 \\
\hline 5468 & C IV & 66 & 19 \\
\hline 5590 & $\mathrm{Ov}$ & 92 & 82 \\
\hline 5803 & C IV & 86 & 190 \\
\hline 6067 & O VIII, O VII & 70 & 14 \\
\hline 6197 & O VI & 71 & 12 \\
\hline 6383: & O III ? & n.m. & n.m. \\
\hline 6436: & He II, O v & n.m. & n.m. \\
\hline 6555 & He II, C IV & 79 & 63 \\
\hline 7060 & C IV & 88 & 44 \\
\hline 7567: & $?$ & n.m. & n.m. \\
\hline 7725 & C IV, O VI & 95 & 158 \\
\hline 8844: & $?$ & n.m. & n.m. \\
\hline 9111: & O III? & n.m. & n.m. \\
\hline
\end{tabular}

of the PN. We identified other faint emission lines of He II, O v, and $\mathrm{O}$ VII (see Table 1 for a list of all the identified features), but some of the faintest ones remained unidentified. We specially noted three emission lines which we identify as O V $\lambda 5590$, O VI

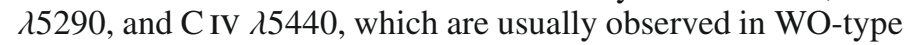
objects. In Fig. 2, we show the LCO spectrum of Th 2-A and identify the emission lines detected in it. We show this spectrum because it covers the largest spectral range.

We performed a quantitative classification of the spectrum of the CSPN of Th 2-A using the criteria described by AN03, based on dereddened line intensities ratios and FWHM of emission lines, we obtained that most of the emission lines strongly indicate a [WO3]-type classification, but the FWHM of the C IV $\lambda$ 5806 is similar to the one measured in [WO4]pec-type stars. Then, we suggest a [WO3]pec spectral classification for the CSPN of Th 2-A. A note of caution has to be added, the line ratios indicate a type between [WO2] and [WO4] and the intrinsic scatter in the classification scheme is also non-negligible as pointed out by AN03.

Th 2-A is an old PN with a dynamical age of 7 kyr estimated in a very simplified way, i.e. ignoring the effects of the velocity gradient and the acceleration over time, and assuming a distance of $2.07 \mathrm{Kpc}$ (Phillips 2004), an angular size of 27!'3 (Tylenda et al. 2003) and an expansion velocity $V_{\exp }=18 \mathrm{~km} \mathrm{~s}^{-1}$ (Meatheringham et al. 1988). The estimations of kinematical ages in PN are still matter of debate (Schonberner et al. 2005), but the classification of the CSPN fits well in the evolution sequence i.e. post-AGB $\rightarrow$ [WC11] to [WC4] $\rightarrow$ [WO4] to [WO1]. Moreover, the hot spectral-type estimated for the CSPN Th 2-A is consistent with the high excitation state of the nebula (Kingsburgh \& Barlow 1994). 

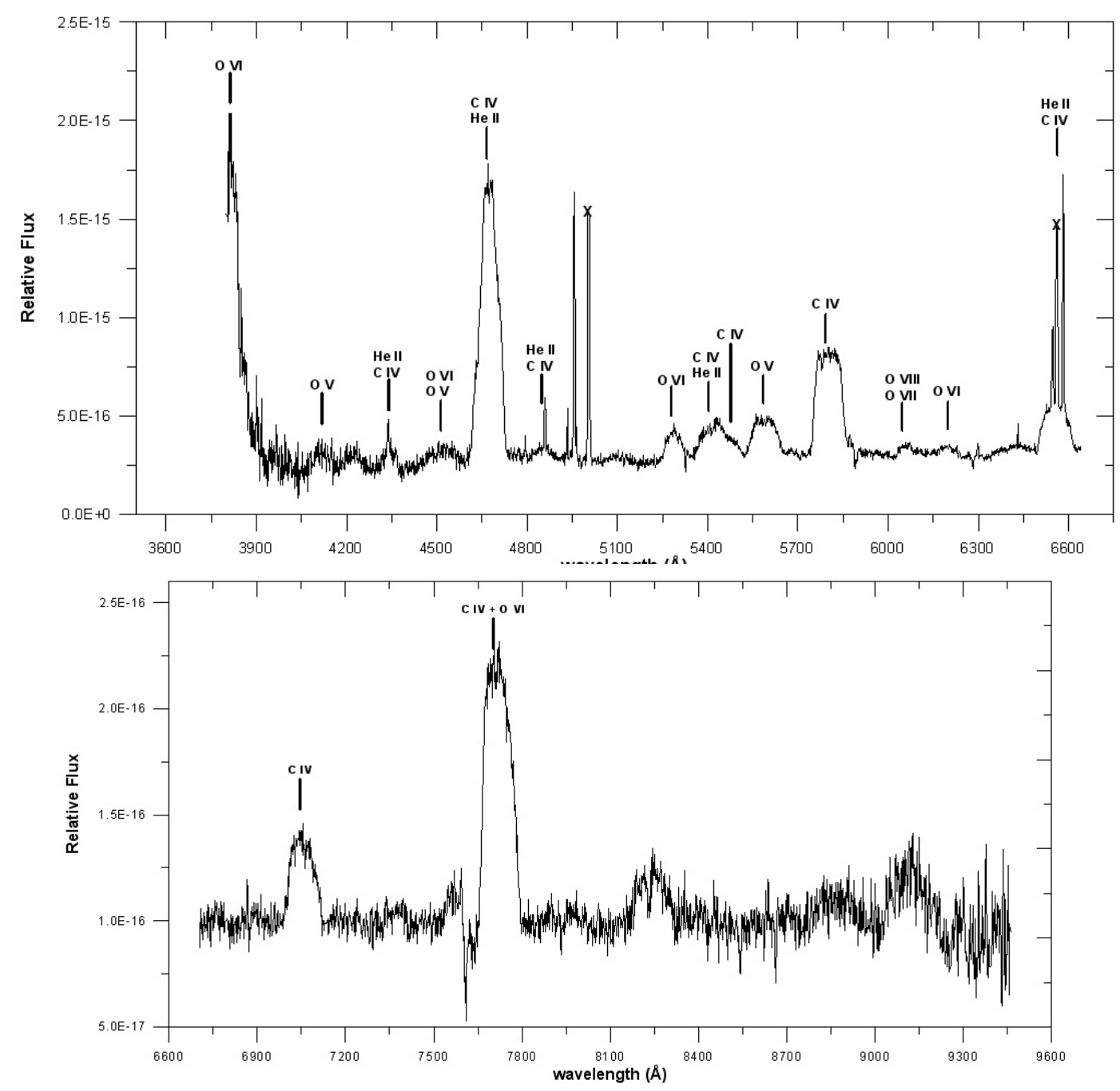

Fig. 2. Blue and red spectra of Th 2-A obtained in December 2007 using LCO. The most intense nebular lines have been truncated ("X"-labeled) in order to show more details of the spectra. Note the broad lines from the central star at $3822 \mathrm{O}$ VI, $4670 \mathrm{C}$ IV and 5805 C IV.

Finally, we want to remark that it is important to perform multi-epoch observations that will help to determine its binary nature (if any). Certainly, there is a surprising lack of surveys that can detect binaries, letting the actual PN binary fraction as unknown, thus some theories that propose that binary interactions have an important role can not be tested (e.g. Moe \& De Marco 2006). Moreover, a full quantitative analysis using stellar atmospheric modeling will be worth while.

Acknowledgements. The CCD and data acquisition system at CASLEO has been financed by R. M. Rich trough U. S. NSF grant AST-90-15827. This work has been partially supported by Concejo de Investigaciones Científicas y Técnicas de la República Argentina (CONICET). We acknowledge Guillermo Bosch for comments that helped to improve the paper, and thank to Nidia Morrell for kindly obtaining a spectrum for us. Some of the data presented in this paper were obtained from the Multimission Archive at the Space Telescope Science Institute (MAST). STScI is operated by the Association of Universities for Research in Astronomy, Inc., under NASA contract NAS5-26555. Support for MAST for non-HST data is provided by the NASA Office of Space Science via grant NAG5-7584 and by other grants and contracts. Based on observations obtained at the Gemini Observatory, which is operated by the Association of Universities for Research in Astronomy, Inc., under a cooperative agreement with the NSF on behalf of the Gemini partnership: the National Science Foundation (United States), the Science and Technology Facilities Council (UK), the National Research Council (Canada), CONICYT (Chile), the Australian
Research Council (Australia), Ministério da Ciência e Tecnologia (Brazil) and Ministerio de Ciencia, Tecnología e Innovación Productiva (Argentina).

\section{References}

Acker, A., \& Neiner, C. 2003, A\&A, 403, 659 (AN03)

Acker, A., Ochsenbein, F., Stenholm, B., et al. 1992, 1996, Strasbourg-ESO catalogue of galactic planetary nebulae, ESO

Barlow, M. J., \& Hummer, D. C. 1982, in Wolf-Rayet Star: Observation, Physics, Evolution, ed. C. W. H. de Loore, \& A. J. Willis (Dordrecht: Reidel), Proc. IAU Symp., 99, 387

Ciardullo, R., Bond, H. E., Sipior, M. S., et al. 1999, AJ, 118, 488

Drew, J. E., Barlow, M. J., Unruh, Y. C., et al. 2004, MNRAS, 351, 206

Gesicki, K., Zijlstra, A. A., Acker, A., et al. 2006, A\&A, 451, 925

Girard, P., Köppen, J., \& Acker, A. 2007, A\&A, 463, 265

van der Hucht, K. A. 2001, New AR, 45, 135

Kerber, F., Mignani, R.P., Guglielmetti, F., \& Wicenec, A. 2003, A\&A 408, 1029 Kingsburgh, R. L., \& Barlow, M. J. 1994, MNRAS, 271, 257

Meatheringham, S. J., Wood, P. R., \& Faulkner, D. J. 1988, ApJ, 334, 862

Moe, M., \& De Marco, O. 2006, ApJ, 650, 916

Phillips, J. P. 2004, MNRAS, 353, 589

Schonberner, D., Jacob, R., \& Steffen, M. 2005, A\&A, 441, 573

Smith, L. F., \& Aller, L. H. 1969, ApJ, 157, 1245

Tylenda, R., Siódmiak, N., Górny, S. K., Corradi, R. L. M., \& Schwarz, H. E. 2003, A\&A, 405, 627 